\title{
Capacidad de discriminación cromática en los procesos de clasificación cerámicos
}

\author{
I. TORTAJADA, B. DEFEZ, G. PERIS-FAJARNÉS, F. BRUSOLA, T. MAGAL \\ Departamento de Ingeniería Gráfica \\ UNIVERSIDAD POLITÉCNICA DE VALENCIA
}

\begin{abstract}
Hoy en día, una gran mayoría de las empresas cerámicas continúan realizando la clasificación de la producción mediante personal especializado (clasificadores) de manera visual. La base del conocimiento que poseen dichos clasificadores proviene de la propia experiencia práctica desarrollada en el puesto de trabajo, no disponiéndose de referencias precisas que marquen procedimientos para usar de manera sistemática herramientas de medida de color, por lo que los criterios utilizados por cada uno de ellos no son los mismos. La evidente mejora que se podría obtener mediante una uniformidad de criterios justifica la búsqueda de procedimientos objetivos para realizar el proceso de clasificación visual.

En este trabajo se proponen criterios homogéneos de clasificación de color para baldosa cerámica. Para ello se plantean una serie de experiencias, realizadas por observadores especializados (clasificadores en activo) y no especializados, a partir de un tipo específico de muestras acromáticas. La capacidad de discriminación de los observadores viene determinada por la ordenación adecuada de las muestras en escalas de grises, estableciendo un procedimiento objetivo para clasificarlas. Además, utilizando ciertas muestras como elementos patrones, se obtiene una agrupación de los estímulos acromáticos que sirve para conocer el intervalo de aceptación en la determinación de la capacidad de discriminación cromática de una población de observadores.
\end{abstract}

Palabras clave: Proceso de clasificación, Inspección visual, Umbral de discriminación de color.

Chromatic discrimination capacity in the ceramics sorting process.

Nowadays, the most of the ceramic tile companies keep on sorting their production visually by means of specialized staff (classifiers). The know how of these classifiers comes from their own practical experience developed in the working place, not having at one's disposal accurate references that indicate the procedures to systematically use tools for color measurement . Therefore, the criteria employed by each of them are not the same. The evident improvement that could be achieved thanks to uniform criteria justifies the search for objective procedures to carry out the visual classification process.

In this paper we propose homogeneous criteria for color classification. In this sense, we put forward a series of experiments, executed by specialized (classifiers) and non-specialized observers, based on a specific set of achromatic samples. The discrimination capacity of the classifiers is determined by the adequate arrangement of the samples according to a greyscale, establishing an objective procedure for their sorting. Moreover, using particular samples as patterns, we obtain a group of the chromatic stimulus that is useful to know the acceptance interval in the determination of the chromatic color-differential thresholds of a set of observers.

Keywords: Sorting Process, Visual inspection, Color-differential thresholds.

\section{EL PROCESO DE CLASIFICACIÓN CERÁMICO}

Tras una década de expansión y liderazgo mundial, el sector productivo de pavimentos y revestimientos cerámicos europeo afronta un futuro incierto, fruto de la apertura de los mercados y la globalización (1). Haciendo frente a este nuevo entorno, la industria cerámica española ha logrado, junto con Italia, alcanzar un liderazgo tecnológico considerable. Sin embargo, algunas fases del proceso productivo continúan siendo fundamentalmente manuales, habiendo variado su práctica muy poco desde su origen (2). Un ejemplo claro lo constituye el proceso de clasificación, como se describe a continuación.
1.1. Proceso de clasificación en la industria de la baldosa cerámica.

En la parte final del proceso productivo de pavimentos y revestimientos cerámicos, las piezas son inspeccionadas para comprobar su resistencia a rotura, escuadrado, calibre y "tono", formando parte todas estas medidas del proceso de clasificación cerámico (3), el cual es necesario realizar antes del embalaje y posterior almacenamiento del producto.

De las cuatro medidas necesarias, las tres primeras solían llevarse a cabo artesanalmente, pero en la actualidad son realizadas exclusivamente de forma mecánica y en algunos 
casos también automática. La clasificación por tonos es el único de estos procesos que todavía puede realizarse de las dos maneras: mediante inspección visual por parte de personal de las propias empresas, y mediante sistemas de inspección automatizados puestos a la salida de horno (4-9).

Cabe destacar que aunque científicamente es incompleto, la utilización del concepto "tono" en el sector cerámico se confunde con el concepto más amplio "color". En este trabajo, se emplea el concepto de "tono" tal y como es usado en la industria, es decir, como la agrupación de baldosas de diferente color realizada por un clasificador, bajo la suposición personal de que pueden ser comercializadas dentro de un mismo grupo (o categoría de producto), debido a que sus "diferencias de color" $(\Delta \mathrm{E})$ son lo suficientemente pequeñas para que no se produzcan reclamaciones en este sentido.

El ojo humano es uno de los mejores medidores de cero que existen (10), no siendo superado por sistemas de inspección automatizados. No obstante, los sistemas de inspección automáticos poseen una serie de ventajas derivadas de la repetibilidad de su funcionamiento al no verse afectados por factores como la fatiga visual, lo que permite mantener constantes los criterios de clasificación aplicados. Por contra, se sabe que las decisiones asociadas a la comparación entre colores realizadas por personas varían a lo largo del tiempo están afectadas por características propias del observador, como pueden ser la edad, el sexo, y el estado psicológico en el que se encuentre $(11,12)$. Además, en procesos de clasificación industriales, a la variabilidad individual hay que añadir las posibles diferencias de criterio entre sujetos.

A pesar de los resultados obtenidos por algunos autores (13-15), que muestran una correlación estadística baja (no mayor de 0.7 ó 0.8 ) entre las diferencias de color percibidas visualmente y las obtenidas aplicando las fórmulas de diferencias de color $\Delta \mathrm{E}_{76}$ y CIE94, se han producido avances en el entendimiento del funcionamiento del sistema visual humano y de sistemas de medida del color que permiten pensar en la posibilidad de establecer un criterio objetivo para la generación de escalas cromáticas $(11,16,17)$ pudiendo aplicar herramientas estadísticas, como el análisis de regresión, para la obtención de tolerancias de aceptabilidad, aplicadas a la industria.

\section{FUNDAMENTO DE LAS EXPERIENCIAS}

Frente a los sistemas de control del color en la cerámica tradicionales (18-21), hoy en día, uno de los sistemas de medida del color más extendidos en la industria es el CIE$\mathrm{L}^{*} \mathrm{a}^{*} \mathrm{~b}^{*}$ (22-30), en donde los tres parámetros que sirven para determinar el color de los objetos a medir son la luminosidad $\left(\mathrm{L}^{*}\right)$, la cantidad de rojo o verde $\left(\mathrm{a}^{*}\right)$ y la cantidad de azul o amarillo $\left(b^{*}\right)$.

En este trabajo se usa el espacio de color CIELab, y se restringen las condiciones experimentales a todos los acromáticos, del blanco al negro, siendo nulos los valores de $a^{*} y b^{*}$. Por ello, la única diferencia entre dos muestras cualesquiera se sitúa en el valor de $L^{*}$.

Partiendo de la generación de una serie uniforme de muestras de gris con valores crecientes de $\mathrm{L}^{*}$, se ha estudiado la evolución en la ordenación realizada por un conjunto de observadores, algunos de los cuales trabajan como clasificadores en industrias de cerámica, mientras que otros no han tenido ningún tipo de experiencia previa en clasificación de color. Se han fijado condiciones usuales de observación, tanto para un grupo como para otro (12).

Dicha ordenación se ha realizado sobre varios conjuntos de muestras, cada uno de los cuales está caracterizado por una diferencia constante en el valor de $L^{*}$ entre las mismas.

A partir de los resultados de esta ordenación se ha pretendido generar un procedimiento que permita realizar una clasificación de las muestras por grupos acorde a ciertos criterios de carácter objetivo.

De acuerdo con esto, puede resultar contradictorio el título del trabajo en relación al contenido experimental del mismo, ya que los componentes cromáticos del espacio de color CIELab son eliminados de la experimentación, quedando tan solo la componente luminosa, siendo quizá más apropiado hablar de discriminación acromática. Sin embargo, dado la complejidad del estudio de la percepción de color, se decidió comenzar las investigaciones variando únicamente uno de los parámetros, dejando la extensión de los otros atributos del color para posteriores trabajos. La elección de la luminosidad responde al hecho de que, industrialmente, la clasificación ejecutada en las empresas cerámicas tiene un carácter fundamentalmente lineal, ordenándose las muestras, a priori, según los tonos sean percibidos como más "claros" o más "oscuros".

\section{GENERACIÓN DE MUESTRAS}

Para la experimental, se preparan una serie de muestras generadas por ordenador, de diferentes niveles de grises, cuyas luminancias varían entre $L^{*} 25$ y $L^{*} 90$ aproximadamente, permaneciendo los valores de $\mathrm{a}^{*} \mathrm{y} \mathrm{b}^{*}$ nulos o próximos a cero.

En total, se elaboraron dos series: una primera serie de 19 muestras con intervalos de $L^{*}$ de 1,1 entre valores de $L^{*}$ de 40 y 60,9; y una segunda serie de 53 muestras con intervalos de $L^{*}$ de 1,2 con valores de $L^{*}$ de entre $L^{*}=27,6$ y $L^{*}=91,2$.

Con ello se pretende cubrir toda la transición acromática del blanco al negro con un número de muestras manejable. Por su parte, los intervalos de $\mathrm{L}^{*}$ vienen condicionados por las tolerancias de los equipos de impresión.

Las muestras, consistentes en una imagen homogénea pegada sobre una base de cartón pluma, subtienden un ángulo de $13^{\circ}$ lo que nos sugiere un tamaño de $12 \times 12 \mathrm{~cm}$. para unas observaciones realizadas aproximadamente a medio metro. Sobre ellas se coloca una lámina de acetato para su protección. La imagen ocupa totalmente la superficie, y el acetato es retirado cuando se realizan las pruebas.

Para la elaboración de todas las imágenes se utiliza el software Adobe Photoshop, que permite calibrar la impresora (HP deskjet 990cxi), que trabaja en modo RGB (genera el gris por mezcla de tres colores: rojo, verde y azul). Además, se genera el perfil de la impresora utilizando el test de calibración RGB Test Chart, para que la impresión obtenida sea lo más aproximada posible al resultado deseado.

Una vez impresas las muestras, se miden los parámetros $L^{*}, a^{*}$ y b* de cada una de ellas con un espectrofotómetro Minolta CM-508i, para asegurar que los valores impresos corresponden con los diseñados y así caracterizarlas perfectamente. Este espectrofotómetro cumple con todos los requisitos especificados por Collaborative Testing Services Inc (CTS), utilizando patrones de medida, cuyas mediciones han sido comparadas entre diferentes laboratorios de prestigio. Es decir, la incertidumbre en los resultados se reduce al nivel de precisión del aparato, y no a errores de medida (31). 


\section{METODOLOGÍA}

Se van a realizar tres tipos de experiencias con diferente número de observadores, empleando las series de muestras comentados en el apartado anterior. A continuación se describe el procedimiento experimental de cada una.

En la primera experiencia se cuenta con un total de 7 clasificadores que trabajan en diferentes empresas cerámicas, realizando su tarea uno a uno de manera independiente. Se les entrega un lote de 19 muestras (tal y como se ha indicado, rectángulos en tonos de gris o acromáticos). Se utilizan condiciones homogéneas para todos los clasificadores en posición y una iluminación de 600 luxes (12). Se les pide que, tras realizar una ordenación de las muestras, de menor a mayor nivel de gris, realicen un agrupamiento de las piezas por lotes tonales o tonos según el criterio utilizado en su trabajo, sin imponerles tiempo límite para ello.

En la segunda experiencia se intenta definir el efecto del entrenamiento. Para ello, se dispone de 6 observadores (no especializados) y 1 clasificador (especializado) que servirá de control. Se les pide que ordenen de menor a mayor valor de $\mathrm{L}^{*}$ varios grupos de muestras como se comenta a continuación, debiendo, en todos los casos, hacerlo en un tiempo limitado, que para cada caso viene en función de la dificultad (menor incremento de $\mathrm{L}^{*}$ entre muestras) y del número de ellas.

Antes de realizar cada una de las experiencias y con cada uno de los observadores, se les pasa un cuestionario con el que se pretende conocer el estado anímico de los mismos, ya que este estado puede influir en experiencias de este tipo (32), de tal forma que, cuando los parámetros indiquen que el estado anímico no es el "normal", entonces, los resultados obtenidos, para ese observador y en esa toma de datos concreta de esa experiencia, sean anulados.

a) En una primera fase se les entrega un conjunto 19 muestras con intervalo de $L^{*}$ de 3,4 unidades de media y con un tiempo límite de 3 minutos.

b) En una segunda fase se les entrega un conjunto 30 muestras con intervalo de $L^{*}$ de 2,2 unidades de media, y se les pide que dicha experiencia la repitan tres veces, teniendo un tiempo límite en cada una de 5 minutos y medio.

c) En una tercera fase se les entrega un conjunto de 53 muestras con intervalo de $L^{*}$ de 1,2 unidades de media, se les pide que dicha experiencia la repitan tres veces. Como el número de muestras era considerable, se decide dividirlas en dos grupos, uno de 27 muestras y otro de 26, teniendo un tiempo límite de 6 minutos y medio para la ordenación de cada uno de ellos.

En la tercera y última experiencia se utilizan 3 clasificadores a los que se les entrega una serie de 19 muestras definidas como tonos patrón con intervalos de $\mathrm{L}^{*}$ medios de 3.4 unidades, y se les pide que agrupen el resto de las muestras por tonos utilizando los tonos de referencia como patrones. También se les indica que pueden generar tonos nuevos si observaban que alguna muestra no puede clasificarse entre las propuestas. En esta tercera experiencia no hay tiempo límite de realización, y no se les hace ninguna indicación respecto de cómo comparar entre muestras de un mismo grupo tonal.

Se les pregunta, al final de cada experiencia, si les parece correcta la propuesta realizada y responden a ello de manera afirmativa.

\section{RESULTADOS Y DISCUSIÓN}

\subsection{Primera experiencia}

Una vez entregadas las 19 muestras al clasificador, éste, sin límite de tiempo debe ordenarlas y agruparlas por tonos según el criterio general que aplica en su trabajo. Las muestras han sido codificadas por la parte posterior con una combinación no seriada de 2 letras, de manera que el observador no pueda identificar la secuencia.

Como se puede observar en la tabla I, el número de grupos tonales o tonos oscila, dependiendo del clasificador, entre 3 y 12 , por lo que no existe un criterio uniforme para la realización de dicho agrupamiento. Se aprecia cómo el número de tonos que se generan es mayor a medida que aumenta el valor de $L^{*}$. Dicho de otra forma, el intervalo de $\mathrm{L}^{*}$ para decidir generar un tono diferente al anterior es menor cuanto más alto es el valor de $L^{*}$.

Del ensayo realizado podríamos destacar las siguientes consideraciones:

1.-No parece existir un criterio homogéneo de clasificación por tonos, dado que la discrepancia es notable en lo que se refiere a decidir por el agrupamiento en "lotes del mismo tono" de las muestras suministradas.

2.- Hay mayores coincidencias para las muestras más oscuras del conjunto de la ordenación, si bien ello puede deberse al hecho de que se generan más grupos tonales con valores de $\mathrm{L}^{*}$ mayores. Para afirmar este hecho sería necesario un mayor número de muestras con intervalos de $\mathrm{L}^{*}$ inferiores a los utilizados.

3.- El número medio de grupos realizados por los clasificadores es de 7,5 grupos para 19 muestras (19 piezas / 7,5 Grupos = Grupos de 2,5 piezas). Ello se corresponde con un intervalo de $L^{*}$ de 2,75 (resultado de multiplicar 2,5 piezas por 1,1 unidades).

TABLA I. CLASIFICACIÓN DE 19 MUESTRAS EN NIVELES DE GRIS CON VALORES DE L* ENTRE 40 Y 60, CON $\triangle$ L* DE 1,1. EN CADA FILA APARECE EL AGRUPAMIENTO POR TONOS DE CADA CLASIFICADOR (UN TOTAL DE 7 CLASIFICADORES). EN CADA COLUMNA APARECE NUMERADA LA IDENTIFICACIÓN DE LA MUESTRA (1 CORRESPONDE A L* DE 40,0 Y 19 CORRESPONDE CON L*=60,9). LA AGRUPACIÓN DE TONOS SE HA REALIZADO POR LETRAS, Y EL NÚMERO TOTAL DE GRUPOS TONALES APARECE EN LA PENÚLTIMA COLUMNA Y EN LA ÚLTIMA EL ÍNDICE DE COINCIDENCIA EN LA CLASIFICACIÓN (ICC).

\begin{tabular}{|c|c|c|c|c|c|c|c|c|c|c|c|c|c|c|c|c|c|c|c|c|c|}
\hline \multirow{2}{*}{ Clasificador } & \multicolumn{19}{|c|}{ AGRUPACIONES TONALES } & \multirow{2}{*}{$\begin{array}{c}\mathrm{n}^{\mathrm{o}} \\
\text { tonos }\end{array}$} & \multirow{2}{*}{ ICC } \\
\hline & 1 & 2 & 3 & 4 & 5 & 6 & 7 & 8 & 9 & 10 & 11 & 12 & 13 & 14 & 15 & 16 & 17 & 18 & 19 & & \\
\hline 1 & a & $\mathrm{a}$ & $\mathrm{a}$ & $\mathrm{a}$ & $\mathrm{b}$ & $\mathrm{b}$ & $\mathrm{c}$ & $\mathrm{c}$ & $\mathrm{d}$ & e & $\mathrm{e}$ & $\mathrm{e}$ & $\mathrm{f}$ & $\mathrm{f}$ & $\mathrm{h}$ & $\mathrm{g}$ & i & $\mathrm{g}$ & $\mathrm{i}$ & 9 & 0.85 \\
\hline 2 & a & a & $\mathrm{a}$ & $\mathrm{a}$ & $\mathrm{a}$ & $\mathrm{a}$ & $\mathrm{b}$ & $\mathrm{b}$ & $\mathrm{b}$ & $\mathrm{b}$ & $\mathrm{b}$ & $\mathrm{b}$ & c & c & c & c & c & c & c & 3 & 0.55 \\
\hline 3 & a & a & $\mathrm{a}$ & $\mathrm{b}$ & $\mathrm{b}$ & $\mathrm{b}$ & $\mathrm{e}$ & $\mathrm{e}$ & $\mathrm{d}$ & $\mathrm{d}$ & c & c & $\bar{f}$ & $\mathrm{f}$ & $\mathrm{h}$ & g & $\mathrm{i}$ & $\mathrm{g}$ & $\mathrm{i}$ & 9 & 0.85 \\
\hline 4 & a & a & a & $\mathrm{a}$ & $\mathrm{a}$ & $\mathrm{b}$ & $\mathrm{C}$ & $\mathrm{d}$ & c & c & c & c & $\mathrm{e}$ & $\mathrm{e}$ & $\mathrm{e}$ & $\mathrm{f}$ & e & e & $\mathrm{e}$ & 6 & 0.85 \\
\hline 5 & a & a & a & $\mathrm{b}$ & $\mathrm{d}$ & c & $\mathrm{e}$ & f & f & g & g & g & $\mathrm{h}$ & $\mathrm{h}$ & $\mathrm{i}$ & i & j & $\mathrm{i}$ & $\mathrm{k}$ & 12 & 0.55 \\
\hline 6 & $\mathrm{a}$ & $\mathrm{a}$ & $\mathrm{a}$ & $\mathrm{a}$ & $\mathrm{b}$ & $\mathrm{b}$ & $\mathrm{d}$ & $\mathrm{c}$ & $\mathrm{c}$ & c & $\mathrm{c}$ & c & e & $\mathrm{e}$ & $\mathrm{g}$ & $\mathrm{f}$ & $\mathrm{g}$ & g & $\mathrm{g}$ & 7 & 0.95 \\
\hline 7 & a & a & a & a & a & $\mathrm{b}$ & c & c & c & $\mathrm{d}$ & $\mathrm{d}$ & d & e & $\mathrm{e}$ & $\mathrm{e}$ & e & $\mathrm{f}$ & e & $\mathrm{f}$ & 6 & 0.85 \\
\hline
\end{tabular}


Se ha definido como medida global para caracterizar el resultado de esta experiencia el "índice de coincidencia en la clasificación" (última columna de la tabla I) que varía entre 0 (ninguna coincidencia) y 1 (totalmente coincidentes). Este índice ha sido obtenido como el valor normalizado de la desviación (diferencia entre el número de tonos creados por cada observador y la media de tonos generada), siendo la fórmula para la normalización (10-d)/10 (33). Como se observa en la tabla, el índice de coincidencia es alto en algunos casos ( 0.95 para el sexto clasificador), pero en general muestra una coincidencia media o baja (valores de 0.85 y 0.55 ).

\subsection{Segunda experiencia}

En la realización de la segunda prueba de ordenación de un lote de 53 piezas con valores de $L^{*}$ que varían entre 27.6 y 91.2 , se aprecia que el comportamiento medio de los observadores no entrenados frente al clasificador es similar. Es decir, no se detecta una diferencia notable entre la capacidad de discriminación de un especialista y el grupo de observadores escogido (deducida del número de errores o porcentaje de errores cometido en la ordenación).

En la primera parte de la prueba, se pide a los observadores realizar la ordenación de 19 muestras entre las que el $\Delta \mathrm{L}^{*}$ es de 3 unidades. La prueba fue realizada de manera correcta por todos los observadores.

En la segunda parte de la experiencia se les pide que realicen dicha ordenación con una serie de 30 muestras con un $\Delta \mathrm{L}^{*}$ medio de 2 unidades. Se observa como se produce un reducido número de errores. La repetición de dicha prueba parece indicar una ligera tendencia a mejorar, quedando confirmada con una tercera repetición, como puede verse en la figura 1 .

Por último, se les pide la realización de la ordenación de una serie de 53 muestras con un $\Delta \mathrm{L}^{*}$ medio de 1 . Los datos recogidos de dicha experiencia muestran la existencia de un efecto derivado del aprendizaje al reflejarse una clara reducción de número de errores cometidos debidos a la repetición de la prueba. Este efecto puede verse claramente en la figura $1 b$.
Por tanto, no parece haber una diferencia manifiesta entre la capacidad de discriminación de tonos entre especialistas y no especialistas, no siendo estos resultados estadísticamente significativos dado que el número de sujetos es demasiado bajo para representar una población estándar. A pesar de ello, la experiencia permite conocer la evolución de los errores de la media de los observadores tomando como referencia los resultados de un clasificador estándar elegido al azar.

A través de las dos gráficas de la figura 1, se observa claramente una tendencia, y es que, el entrenamiento planeado mejora la capacidad de discriminación por parte de todos los observadores, tanto especialistas como no especialistas.

\subsection{Tercera experiencia}

Se realiza la experiencia con 3 clasificadores y se les aconseja explícitamente que si alguna(s) de las muestras no encaja(n), según su criterio, en alguno de los grupos, consideren que pueden generar un nuevo tono (o los tonos necesarios), e intenten reagrupar aquellas que falten por encajar.

La propuesta de clasificación de tonos fue considerada como válida por los 3 observadores.

Cómo se puede ver en la tabla II, únicamente uno de los observadores consideró necesario realizar un nuevo tono.

Cabe decir que en algunas de las agrupaciones no coincidía el número de muestras (ni el tipo particular de las mismas) agrupadas en distintos tonos, para cada uno de los observadores.

De este experimento se deduce que se puede imponer a los clasificadores un criterio de clasificación a partir de determinados tonos de referencia, lo cual es un principio de metodología objetiva. El desarrollo completo de esta metodología podría resolver una gran parte de los problemas actuales de control de tono, ya que el principal obstáculo al que se enfrentan los profesionales es la no existencia de referencias.

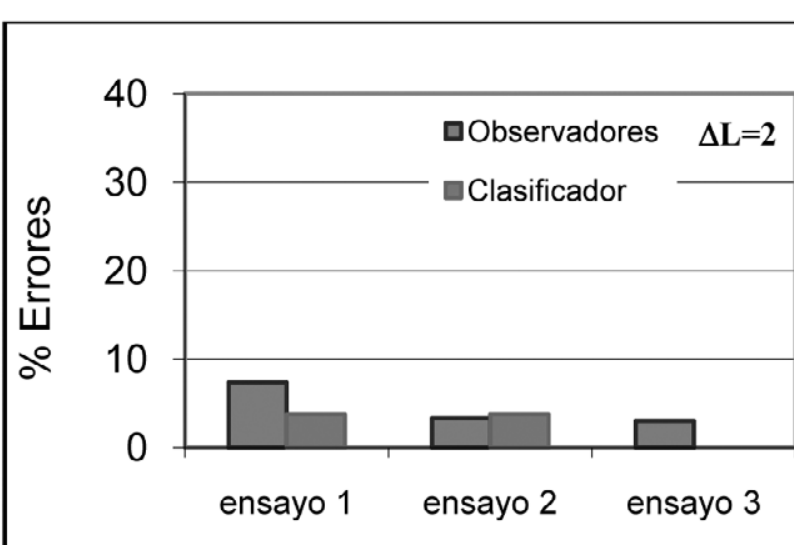

(1a)

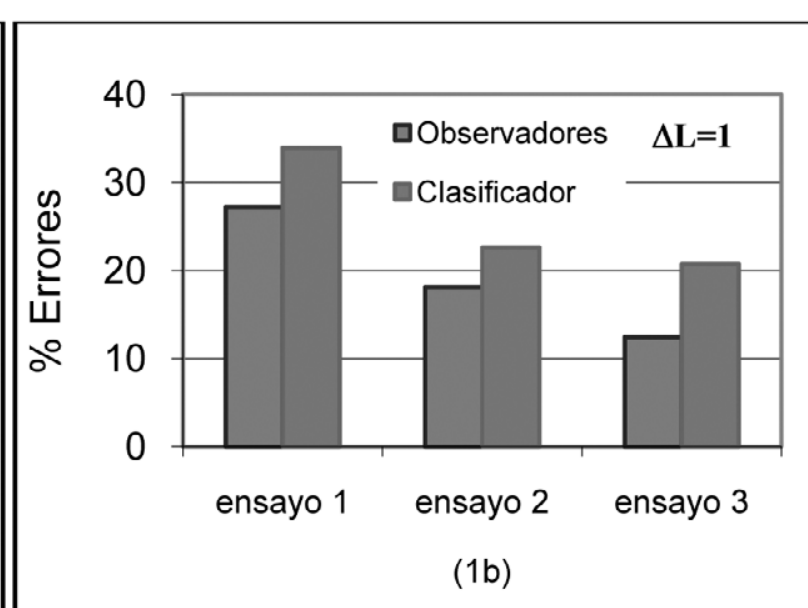

Fig. 1- Evolución de los errores con el número de ensayos. Se representa el número medio de errores cometidos por un conjunto de 6 observadores, y el cometido por una persona de clasificción de la industria cerámica. Las gráficas indican la evolución de dicho porcentaje de error tras la realización de una, dos o tres repeticiones que consisten en ordenar una serie de muestras acromáticas con intervalos de $\mathrm{L}^{*}$ medios de 2 (1a) y de 1 (1b). 
TABLA II. AGRUPACIÓN DE LAS DISTINTAS MUESTRAS ALREDEDOR DE LOS PATRONES CONSIDERADOS COMO PATRONES CON $\triangle L^{*}$ DE 3.4 UNIDADES, OBTENIDA POR LOS 3 CLASIFICADORES DE LA EXPERIENCIA 3. SEÑALAR QUE EL CLASIFICADOR 1 DECIDE GENERAR UN NUEVO TONO PATRÓN (PIEZAS 34-35-36).

\begin{tabular}{|c|c|c|c|}
\hline \multirow{2}{*}{ muestras } & \multicolumn{3}{|c|}{ clasificadores } \\
\hline & Clasif. 1 & Clasif. 2 & Clasif. 3 \\
\hline 1 & $\mathrm{a}$ & $\mathrm{a}$ & a \\
\hline 2 & a & $\mathrm{a}$ & $\mathrm{a}$ \\
\hline 3 & $\mathrm{a}$ & $\mathrm{a}$ & $\mathrm{a}$ \\
\hline 4 & $\mathrm{~b}$ & $\mathrm{a}$ & $\mathrm{b}$ \\
\hline 5 & $\mathrm{~b}$ & $\mathrm{~b}$ & $\mathrm{~b}$ \\
\hline 6 & $\mathrm{~b}$ & $\mathrm{~b}$ & c \\
\hline 7 & c & $\mathrm{b}$ & c \\
\hline 8 & c & $\mathrm{c}$ & $\mathrm{c}$ \\
\hline 9 & c & c & $\mathrm{d}$ \\
\hline 10 & d & c & d \\
\hline 11 & d & d & $\mathrm{e}$ \\
\hline 12 & $\mathrm{~d}$ & $\mathrm{~d}$ & e \\
\hline 13 & e & d & e \\
\hline 14 & $\mathrm{e}$ & e & $\mathrm{f}$ \\
\hline 15 & $\mathrm{e}$ & e & $\mathrm{f}$ \\
\hline 16 & $\mathrm{f}$ & $\mathrm{f}$ & f \\
\hline 17 & $\mathrm{f}$ & $\mathrm{f}$ & g \\
\hline 18 & $\mathrm{~g}$ & $\mathrm{f}$ & g \\
\hline 19 & $\mathrm{~g}$ & g & g \\
\hline 20 & $\mathrm{~g}$ & g & $\mathrm{h}$ \\
\hline 21 & $\mathrm{~h}$ & g & $\mathrm{h}$ \\
\hline 22 & $\mathrm{~h}$ & $\mathrm{~h}$ & $\mathrm{~h}$ \\
\hline 23 & $\mathrm{~h}$ & $\mathrm{~h}$ & $\mathrm{i}$ \\
\hline 24 & $\mathrm{i}$ & $\mathrm{i}$ & $\mathrm{i}$ \\
\hline 25 & i & $\mathrm{i}$ & $\mathrm{i}$ \\
\hline 26 & $\mathrm{i}$ & $\mathrm{i}$ & $\mathrm{j}$ \\
\hline 27 & $\mathrm{j}$ & $\mathrm{j}$ & $\mathrm{j}$ \\
\hline 28 & j & j & j \\
\hline 29 & $\mathrm{j}$ & $\mathrm{j}$ & $\mathrm{k}$ \\
\hline 30 & $\mathrm{k}$ & $\mathrm{k}$ & $\mathrm{k}$ \\
\hline 31 & $\mathrm{k}$ & $\mathrm{k}$ & $\mathrm{k}$ \\
\hline 32 & 1 & $\mathrm{k}$ & 1 \\
\hline 33 & 1 & 1 & 1 \\
\hline 34 & $x$ & 1 & 1 \\
\hline 35 & $x$ & 1 & $\mathrm{~m}$ \\
\hline 36 & $x$ & $\mathrm{~m}$ & $\mathrm{~m}$ \\
\hline 37 & $\mathrm{~m}$ & $\mathrm{~m}$ & $\mathrm{~m}$ \\
\hline 38 & $\mathrm{~m}$ & $\mathrm{~m}$ & $\mathrm{n}$ \\
\hline 39 & $\mathrm{~m}$ & $\mathrm{n}$ & $\mathrm{n}$ \\
\hline 40 & $\mathrm{n}$ & $\mathrm{n}$ & $\mathrm{n}$ \\
\hline 41 & $\mathrm{n}$ & $\tilde{\mathrm{n}}$ & $\tilde{\mathrm{n}}$ \\
\hline 42 & $\mathrm{n}$ & $\tilde{\mathrm{n}}$ & $\tilde{n}$ \\
\hline 43 & $\tilde{\mathrm{n}}$ & $\tilde{\mathrm{n}}$ & $\tilde{\mathrm{n}}$ \\
\hline 44 & $\tilde{\mathrm{n}}$ & $\mathrm{o}$ & o \\
\hline 45 & $\tilde{\mathrm{n}}$ & $\mathrm{o}$ & $\mathrm{o}$ \\
\hline 46 & o & $\mathrm{p}$ & o \\
\hline 47 & o & $\mathrm{P}$ & o \\
\hline 48 & $\mathrm{o}$ & $\mathrm{p}$ & $\mathrm{p}$ \\
\hline 49 & o & $\mathrm{p}$ & $\mathrm{p}$ \\
\hline 50 & $\mathrm{p}$ & $\mathrm{p}$ & $\mathrm{p}$ \\
\hline 51 & $\mathrm{p}$ & $\mathrm{q}$ & $\mathrm{p}$ \\
\hline 52 & $q$ & $\mathrm{q}$ & $\mathrm{q}$ \\
\hline 53 & $\mathrm{q}$ & $\mathrm{q}$ & $\mathrm{q}$ \\
\hline
\end{tabular}

\section{CONCLUSIONES}

Una vez finalizada la experiencia, se pueden extraer varias conclusiones muy interesantes para el sector.

Por un lado, no existe un criterio homogéneo de clasificación por tonos dentro de la industria cerámica, dado que la discrepancia es notable en lo que se refiere a decidir por el agrupamiento en "lotes del mismo tono" de las muestras suministradas.

Tampoco hay una diferencia manifiesta entre la capacidad de discriminación de tonos entre especialistas y no especialistas, observándose que el entrenamiento planeado mejora la capacidad de discriminación por parte de todos los observadores.

Por otro lado, el estudio concreto de los resultados del experimento apunta una tendencia al agrupamiento por grupos tonales con $\Delta \mathrm{L}^{*}$ medios de 3 unidades, si bien dicho valor medio debe definirse por tramos, de forma que se puede aumentar para tonos claros y disminuir para tonos oscuros.

Finalmente, destaca que es posible, mediante la realización de estudios posteriores, obtener una mayor uniformidad en los criterios de clasificación mediante un entrenamiento orientado de los observadores. Complementariamente, es factible obtener un criterio que permita fijar un $\Delta \mathrm{L}^{*}$ mediante el estudio de capacidad de discriminación de una población de observadores. Dicho incremento se podría definir como aquel que un cierto porcentaje de observadores especializados confundiría o agruparía dentro de un mismo grupo tonal.

\section{LINEAS FUTURAS DE INVESTIGACIÓN}

Tras el análisis de las conclusiones, se plantean varias líneas futuras de trabajo.

Por un lado, sería conveniente realizar las experiencias añadiendo al mismo tipo de muestras los atributos de tono y saturación (valores de "a" y "b" distintos de cero). Esto permitiría una evaluación cromática en toda su extensión.

Por otro lado, cambiar el material de las muestras usando baldosas reales y comparando la coincidencia en la clasificación de distintos clasificadores, tal y como se hizo para la industria de la madera (34) nos permitiría validar nuestro principio de metodología en condiciones realistas. La comparación de la clasificación humana frente a los sistemas automatizados, también es una línea de trabajo a continuar.

\section{BIBLIOGRAFÍA}

1. E. Criado, E. Sánchez, M. Regeiro, La industria cerámica española, ¿ante un cambio de ciclo?, Bol. Soc. Esp. Ceram. V., 43, 1, 85-101, (2004).

2. J. Albors, J.L. Hervás, La industria cerámica europea en el siglo XXI. Retos tecnológicos y desafíos de la próxima década, Bol. Soc. Esp. Ceram. V., 45, 1, 13-21, (2006).

3. I. Tortajada, G. Peris-Fajarnés, M. Aguilar, P. Latorre, Análisis del proceso de clasificación cerámico Bol. Soc. Esp. Ceram. V., 45, 1, 22-27, (2006).

4. R. Massen, Clasificación óptica automática de azulejos porcelanados, Cerámica Información, 252, 29-32, (2000).

5. R. Massen, T. Franz, La calidad de los sistemas automáticos de inspección de azulejos, Cerámica Información, 273, 63-66, (2001).

6. F. López, J. M. Valiente, J. R. Navarro, F. Acebrón, Inspección automática de tonalidad en procesos industriales: aplicación a la clasificación de azulejos, XIV Congreso Internacional de Ingeniería Gráfica, 288-294, Santander (2002).

7. J. M. Valiente, F. Acebrón ,F. López, J. R. Navarro, Sistema de inspección visual automática de azulejos de patrón fijo, XIV Congreso Internacional de Ingeniería Gráfica, 309-318, Santander (2002).

8. R. Massen, Aumento de la producción cerámica con sistemas de visión 
bivalentes: inspección y seguimiento del proceso automático con la misma tecnología, QUALICER 2004, 3-6, España.

9. Proyecto Europeo MONOTONE (Quality Control for Industrial Printing), Documento de trabajo V Programa Marco Unión Europea, (2003), G1RDCT-2002-00783.

10. P. Capilla, J. M. Artigas, A. Felipe, A. Pujol, Óptica Fisiológica: Psicofísica de la visión, Ed.: McGraw-Hill Interamericana, ISBN 84-486-0115-7, Madrid, 1995.

11. Y. S. W. Li, C. W. M. Yuen, K. W. Yeung, K. M. Sin, Prediction of the best-fit regression model to correlate instrumental colour measurement and visual assessment, J. Soc. Dyers Colour., 115, 22-31, (1999).

12. E. Hita, J. Romero, Análisis de la influencia de las condiciones de observación en los procesos de discriminación en color, Op. Pur. Apl., 14, 11-17, (1981).

13. R. McDonald, A review of the relationship between visual and instrumental assessment of colour difference, part 1, J. Oil Colour Chem. Assoc. 65, 43-53, (1982).

14. R. McDonald, A review of the relationship between visual and instrumental assessment of color difference, part 2, J. Oil Colour Chem. Assoc., 65, 93-106, (1982).

15. K. Witt, Modified CIELAB formula tested using a textile pass / fail data set, Color Res. Appl. 19, 273-276, (1994).

16. Y. S. W. Li, C. W. M. Yuen, K. W. Yeung, K. M. Sin, Regression analysis to determine the optimum colour tolerance level for instrumental shade sorting, J. Soc. Dyers Colour., 115, 95-99, (1999).

17. C. Alder, K.P. Chaing, T.F. Chong, E, Coates, A.A. Khalili, B. Rigg, Uniform chromaticity scales - New experimental data, J. Soc. Dyers Colour., 98, 14, (1982).

18. R. W. Batchelor, La medida del color y su aplicación a la industria cerámica, Bol. Soc. Esp. Ceram. V., 4, 5, 441-456, (1965).

19. J. Jonckheere, Colorimétrie Industrielle, Bull. Soc. Franç. Céram.,76, 3, 89-95, (1967).

20. J. Vilnat, M. Pagano, Essais et mesures colorimétriques en céramique, Bull. Soc. Franç. Céram., 76, 3, 97-119, (1967).
21. J. Gileod, La mesure de la couleur et ses applications dans l'industrie, Bull. Soc. Franç. Céram., 83, 2, 69-77, (1969).

22. R.E. Gago, Colorimetría espacio uniforme, Bol. Soc. Esp. Ceram. V, 15, 6, 363-370 (1976)

23. R. W. Harold, Colour technology in the textile industry, Research Triangle Park, NC : AATCC, 155, (1983).

24. R. C. Kuehni, Colour technology in the textile industry, Research Triangle Park, NC : AATCC, 123, (1983).

25. J.M. Artigas, J.C. Gil, A. Felipe, El espacio uniforme de color CIElab. Utilización. Rev. Agroquím Tecnol Alimen., 25, 3, 316-320, (1985).

26. R. G. Kuehni, Industrial Color Difference: Progress and Problems, Color Res. Appl., 15, 5, 261-265, (1990).

27. R. MacDonald, European Practices and Philosophy in Industrial Colourdifference Evaluation, Color Res. Appl., 15, 5, 249-260, (1990).

28. M. Melgosa, E. Hita, J. Romero, L Jiménez del Barco, Color-Discrimination Thresholds translated from the CIE $(x, y, Y)$ Space to the CIE $1976\left(\mathrm{~L}^{*}, \mathrm{a}^{*}, \mathrm{~b}^{*}\right)$, Color Res. Appl., 19, 1, 10-18, (1994).

29. D. H. Alman, CIE Technical Comité 1-29, Industrial Color-Difference Evaluation, Color Res. Appl., 16, 3, 219-220, (1991).

30. K. Witt, CIE Guidelines for Coordinated Future Work on Industrial ColourDifference Evaluation, Color Res. Appl., 20, 6, 399-403, (1995).

31. J. Campos, E. Hita, J. Romero, M. Melgosa, J.M. Artigas, P. Capilla, A. Felipe, F.M. Verdú, J. Pujol, I. Negueruela, L. Jiménez del Barco, Avances y tendencias recientes en colorimetría, Op. Pur. Apl., 30, 1-35 (1997).

32. Fabio G. Melchiades, Anselmo O. Boschi, Cores e tonalidades em revestimentos cerâmicos, Cerámica Industrial, 4, 1-6, (1999).

33. I. Tortajada, V. Blanca, M. Martínez, M. Aguilar, Variation with the contrast and modulation ratio of an achromatic grating on view of a chromatic periodic test, The $10^{\text {th }}$ Congress of the International Colour Association (AIC Colour 2005), Granada (España).

34. H.A. Huber, C.W. McMillin, J.P. Mckinney, Lumber defect detection abilities of furniture rough mill employees, Forest Products Journal, 35, 79-82 (1985)

Recibido: 05.11 .07

Aceptado: 31.03 .08 